\title{
Preface to the Special Issue on Paleopathology
}

\author{
Hisashi FuJITA ${ }^{1 *}$, Dong Hoon $\mathrm{SHIN}^{2 * *}$ \\ ${ }^{1}$ Department of Bioanthropology, Niigata College of Nursing, Joetsu 943-0147, Japan \\ ${ }^{2}$ Bioanthropology and Paleopathology Lab, Department of Anatomy/Institute of Forensic Science, Seoul National University College
} of Medicine, Seoul, South Korea

Notwithstanding the significant anthropological advancements that have taken place over the past several decades, the health and disease statuses of ancient people have yet to be fully elucidated. To this end, paleopathology - the pathological study of tissues remnant in ancient biological specimens - has been established by many pioneering researchers around the world. The field includes various sub-disciplines such as the diagnosis of specific diseases seen in archaeological specimens, the tracing of secular changes in health and disease patterns of human populations, and the biological reconstruction of pathogenic evolution by molecular techniques. The information obtained inspires ever-greater achievements, thereby contributing to the comprehensive understanding of the reciprocal interaction between human beings and disease throughout history.

Anthropological Science $(A S)$ is an authoritative academic journal with a long publishing history, some issues of which have already included paleopathology-related articles. Nonetheless, the forums and opportunities for paleopathologists to discuss their works in this journal are unfortunately still insufficient relative to the recent explosion of new findings in the field. The current special issue of $A S$, which is devoted exclusively to paleopathology, is therefore indeed very timely and meaningful to concerned researchers.

The contributors to this special issue of $A S$ discuss recent global paleopathological trends - the various scientific techniques employed, the research outcomes, and their interpretation. Some authors have found important clues to the health and disease statuses of ancient societies, based in particular on osteology, one of the most convenient and efficient approaches to the study of ancient human populations.

Jung and Woo's osteoarchaeological report touches on the long-standing debate among Korean historians and anthropologists about the artificially deformed crania of some ancient Yean-ri skeletons. As the authors point out, early historical documents describe in detail how cranial deformities were made by compressing the neonate's head with a stone, resulting in vault flattening along the sagittal plane with ex-

\footnotetext{
* Correspondence to: Hisashi Fujita, Department of Bioanthropology, Niigata College of Nursing, 240 Shinnan, Joetsu 943-0147, Japan. E-mail: hfujita@niigata-cn.ac.jp

** Correspondence to: Dong Hoon Shin, Department of Anatomy/ Institute of Forensic Science, Seoul National University College of Medicine, Seoul, South Korea

E-mail: cuteminjae@gmail.com

Published online 7 April 2017

in J-STAGE (www.jstage.jst.go.jp) DOI: 10.1537/ase.170224
}

pansion of the mediolateral dimensions. The authors nevertheless felt keenly the lack of additional cranial deformity cases among other ancient skeletal collections maintained in South Korea. They therefore first revisited the cases from the Yean-ri site before comparing them with Nukdo and Imdang skulls of the same historical period.

Meanwhile, paleoparasitology, which entails the identification and subsequent microscopic or molecular analysis of ancient parasite species in archaeological samples, has an important bearing on the entire field of paleopathology. In this special issue, Seo et al. analyze coprolites removed from Joseon-period mummies, adding new paleoparasitological outcomes to the existing pool of data already maintained in South Korea. These results will provide invaluable insights into the parasitism of pre-industrial East Asian societies that are unobtainable by conventional historical investigation.

Using a combined historical, archaeo-anthropological and paleo-immunological approach, Cesana et al. reconstruct the origin and early spread of the Black Death (1347-1348 CE) from one of the original epicenters, Genoa (Liguria, northern Italy). A mass grave, archeologically dated to the second half of the 14th century, was uncovered in the mountain cemetery of San Nicolao di Pietra Colice near Genoa. Stratigraphy indicated that the individuals buried there had died either simultaneously or within a very short span of time. Given the time frame and the absence of any signs of violence, Yersinia pestis was posited to have been the cause of death. To strengthen their hypothesis, Cesana et al. tested the skeletal remains with the rapid diagnostic test for plague, which confirmed the presence of the $Y$. pestis-specific F1 antigen. So far, theirs is the first report focusing on the biological identification of that antigen in an Italian settlement dating to the time of the documented arrival of the Black Death.

Another interesting report in this issue is a paleoradiological study by Piombino-Mascali, Zink, and Panzer on the Piraino mummies of Italy. As the mummies were completely clothed, and their transport outside the crypt was not permitted, the only way to study them was by in situ radiological analysis. In their report, the authors clearly demonstrate the special utility that radiological analysis offers for the paleopathological study of ancient or modern mummies.

One of the main objectives of paleopathology today is to contribute to modern medicine by precise and thorough examination and comparison of public health and nutrition statuses between ancient and modern populations. Fujita and Adachi, having conducted a study on a skull from ancient Egypt from the University of Cambridge, found that ancient 
Egyptians had high frequencies of certain stress markers and dental caries indicative of a nutritious diet and a healthy lifestyle. Indeed, if their living conditions were to be proved superior to those of today's Nigerians and Somalis, research of this type could have a substantially beneficial impact not only on anthropology but also on the quality of current medical care. Such research, furthermore, will perhaps pave the way for future paleopathology.

This special issue evolved out of exchanges between Jap- anese and South Korean researchers at the 'Korea-Japan Forum on Paleopathology in Asia' convened in Busan in 2013. Friendly and fruitful relationships are being built not only between Japan and South Korea but also among many nations' paleopathology researchers. It is our tremendous pleasure to see the international development of paleopathology within the wider field of biological anthropology continue in this special issue of $A S$. 